\title{
CONTROL OF BIFURCATION STRUCTURES USING SHAPE OPTIMIZATION*
}

\author{
NICOLAS BOULLÉ ${ }^{\dagger}$, PATRICK E. FARRELL ${ }^{\dagger}$, AND ALBERTO PAGANINI ${ }^{\ddagger}$
}

\begin{abstract}
Many problems in engineering can be understood as controlling the bifurcation structure of a given device. For example, one may wish to delay the onset of instability or bring forward a bifurcation to enable rapid switching between states. We propose a numerical technique for controlling the bifurcation diagram of a nonlinear partial differential equation by varying the shape of the domain. Specifically, we are able to delay or advance a given branch point to a target parameter value. The algorithm consists of solving a shape optimization problem constrained by an augmented system of equations, the Moore-Spence system, that characterize the location of the branch points. Numerical experiments on the Allen-Cahn, Navier-Stokes, and hyperelasticity equations demonstrate the effectiveness of this technique in a wide range of settings.
\end{abstract}

Key words. bifurcation analysis, shape optimization, Moore-Spence system

AMS subject classifications. 65P30, 65P40, 37M20, 65K10

DOI. $10.1137 / 21 \mathrm{M} 1418708$

1. Introduction. A striking property of nonlinear partial differential equations (PDEs) is that they may support multiple solutions not trivially related via the nullspace of a linear operator. These solutions are captured in a bifurcation diagram, recording the solutions $u \in U$ to an equation

$$
F(u, \lambda)=0
$$

as a parameter $\lambda \in \mathbb{R}$ is varied. Here, $U$ is a Banach space of functions defined on a bounded domain $\Omega \subset \mathbb{R}^{d}, d \in \mathbb{N}_{+}$, and $F: U \times \mathbb{R} \rightarrow U^{\star}$, where $U^{*}$ denotes the dual space of $U .{ }^{1}$ Throughout the paper, we assume that the nonlinear operator $F$ is Fréchet differentiable, i.e., $F \in C^{1}\left(U \times \mathbb{R}, U^{*}\right)$. When a system is to be designed, consideration of multiple solutions can be of primary importance: for example, one may wish to ensure that an aircraft remains in a high-lift rather than low-lift regime in takeoff [43] or may wish to exploit bistability in the design of a liquid crystal display that only consumes power when an image is changed [42].

* Submitted to the journal's Methods and Algorithms for Scientific Computing section June 1, 2021; accepted for publication (in revised form) September 21, 2021; published electronically January $5,2022$.

https://doi.org/10.1137/21M1418708

Funding: The work of the first author was supported by the EPSRC Centre for Doctoral Training in Industrially Focused Mathematical Modelling (EP/L015803/1) in collaboration with Simula Research Laboratory. The work of the second author was supported by EPSRC grants $\mathrm{EP} / \mathrm{V} 001493 / 1$ and EP/R029423/1.

${ }^{\dagger}$ Mathematical Institute, University of Oxford, Oxford, OX2 6GG, UK (boulle@maths.ox.ac.uk, patrick.farrell@maths.ox.ac.uk).

${ }^{\ddagger}$ School of Mathematics and Actuarial Science, University of Leicester, Leicester, LE1 7RH, UK (a.paganini@leicester.ac.uk)

${ }^{1}$ This is a convenient way of writing variational problems. The variational problem

$$
\text { find } u \in U \text { such that } G(u, \lambda ; v)=0 \text { for all } v \in U
$$

may be recast as (1.1) by defining

$$
\langle F(u, \lambda), v\rangle:=G(u, \lambda ; v)
$$


We consider the mathematical control of the bifurcation structure of a physical system and formulate an algorithm that modifies the domain $\Omega$ on which a PDE is posed in order to advance or delay a single specified bifurcation point. Several engineering problems may be understood as such a task. For instance, in wing design one typically aims to avoid instabilities due to the buckling of the wing structure. In this example, the bifurcation parameter $\lambda$ is the scaling of the load applied to the wing. To avoid buckling, one can modify the design of the structure $\Omega$ to delay the first branch point (with respect to $\lambda$ ) and thus remove potentially unstable solutions. A similar approach might be taken to the design of aircraft stiffeners [77] and other components. On the contrary, in other applications, inducing new branches might be desirable. For instance, this is the case in the design of new mechanical metamaterials that exploit instabilities to rapidly switch between configurations, as in the snapping of a Venus flytrap [28, 47, 71].

Both bifurcation analysis and PDE-constrained optimization are now mature fields, but their combination has essentially been explored, in a number of related works, through indirect methods that exploit prior model information in specific cases. As an example, Penzler, Rumpf, and Wirth [56] performed shape optimization in the context of nonlinear elasticity and had to design a robust minimization method to handle non-uniqueness of the solution arising from buckling instabilities. More recently, Thomsen, Wang, and Sigmund [68] considered a topology optimization problem to maximize strength of materials against buckling instabilities, while the control of flow bifurcations in channels using shape optimization was analyzed in [72]. In both cases, the state equations and corresponding eigenvalue problems for finding the critical modes are decoupled in the inner loop of the optimization algorithm to reduce the implementation complexity. Finally, a direct method for performing shape optimization of buckling structures using a characterization of critical points was proposed in [59]. The algorithm optimizes the critical load factor over some shape parameters variables using sequential quadratic programming methods [51, Chap. 18], and the underlying system of equations is solved using a penalty formulation to avoid convergence issues near critical points.

In this work, we formulate the task of controlling an isolated branch point as a PDE-constrained shape optimization problem. The essential idea of the formulation is to characterize the branch point as the solution of an augmented system of equations. There are many choices for how to describe branch points in this way (see, e.g., [63, sect. 5.4]). In our case we choose the system proposed by Seydel [62] and Moore and Spence [49], referred to as the Moore-Spence system. We then propose a generic numerical algorithm for solving the constrained optimization problem. This is a rather challenging task: none of the state equation (1.1), the Moore-Spence system, or the outer shape optimization problem have unique solutions.

Much of the literature on PDE-constrained optimization requires that the controlto-state map is single-valued $[2,17,35,39,65,69]$. In fact, in many analyses some smoothness of the map is further required. However, the case where the implicit function theorem cannot be applied has also been considered in both finite and infinite dimensions [18, 36, 37]. Some experiments involving multiple solutions of optimal control problems, arising from multiple solutions of the underlying control-to-state map, were recently presented in [57]. In our work, we address the challenges posed by the co-existence of multiple solutions by careful selection of the initial guesses employed and their updates, which occur while the optimization is carried out, to exploit local uniqueness of isolated branch points. Our algorithm proves successful in several numerical experiments and allows us to find a new shape $\Omega^{\star}$ for which a 
branch of solutions arises at a specific value $\lambda=\lambda^{\star}$. Additionally, we release an open-source implementation of the algorithm built on Firedrake [58], PETSc [7], and Fireshape [54].

While this paper focuses on controlling simple pitchfork and fold bifurcation points with respect to the shape of the domain, we expect that the ideas developed here also apply to other settings, such as the control of the bifurcation diagram with respect to a parameter $\lambda_{1} \in \mathbb{R}$ as another parameter $\lambda_{2}$ in some (possibly infinitedimensional) parameter space is varied, or the control of other kinds of bifurcations such as Hopf bifurcations.

The paper is organized as follows. We first review the characterization we use of branch points using augmented systems of PDEs in section 2. Then, in section 3, we describe an algorithm for performing PDE-constrained shape optimization constrained by the augmented system. In section 4 , we combine these numerical techniques in an algorithm that modifies a shape to delay or advance a given branch point. Finally, in section 5, we apply this technique to delay the bifurcations of three numerical examples involving the Allen-Cahn equation, the Navier-Stokes equations, and the compressible hyperelasticity equation.

2. Characterization of branch points. Bifurcation diagrams of problems of the form $F(u, \lambda)=0$ may contain branch points (see [63]), which are characterized by the change in the number of solutions as $\lambda$ varies in a small interval around $\lambda^{\star}$. This implies that the Fréchet derivative $F_{u}\left(u^{\star}, \lambda^{\star}\right)$ with respect to the variable $u$ at the branch point $\left(u^{\star}, \lambda^{\star}\right)$ cannot be an isomorphism (otherwise, by the implicit function theorem, there would exist locally a unique solution curve $(\lambda, u(\lambda))$, but this is not compatible with Definition 2.1 below). Therefore, we are interested in the computation of singular points, i.e., solution pairs $\left(u^{\star}, \lambda^{\star}\right)$ to (1.1) such that $F_{u}\left(u^{\star}, \lambda^{\star}\right)$ does not have a bounded inverse.

The nonlinear operator $F$ may have a symmetry group $\mathcal{G}$ acting on $U$ such that for all group elements $g \in \mathcal{G}$ and $\lambda \in \mathbb{R}, F(u, \lambda)=0 \Longleftrightarrow F(g \cdot u, \lambda)=0$. Here $g \cdot u$ represents the action of the element $g$ on the solution $u$. Such a group is called a Lie group when it has a structure of a smooth manifold and when the group multiplication and inversion operations are smooth maps [32, 52]. In the presence of such symmetries, one is typically interested in points at which the number of distinct group orbits varies, which are sets of the form: $\mathcal{G}(u)=\{g \cdot u: g \in \mathcal{G}\}$. In this work, we assume that no such Lie groups exist for $F$ so that we may consider distinct solutions rather than distinct group orbits.

DEFINITION 2.1 (branch point). Assume that the operator $F$ does not have a Lie group of symmetries. For $\lambda \in \mathbb{R}$, define $\mathbf{U}_{\lambda}:=\{u \in U: F(u, \lambda)=0\}$, and, for $u^{\star} \in U$ and $\epsilon>0$, let $B_{\epsilon}\left(u^{\star}\right):=\left\{u \in U:\left\|u^{\star}-u\right\|<\epsilon\right\}$. A branch point (also called a bifurcation point) with respect to $\lambda$ is a solution pair $\left(u^{\star}, \lambda^{\star}\right)$ to $F(u, \lambda)=0$ such that, for any $\varepsilon>0$, the cardinality of $\mathbf{U}_{\lambda} \cap B_{\varepsilon}\left(u^{\star}\right)$ changes when $\lambda$ passes $\lambda^{\star}$.

We illustrate some different situations with branch points arising in the bifurcation diagram of a nonlinear PDE in Figure 1. Figure 1(a) features a fold bifurcation with a turning point. Figure 1(b) and (c) illustrate two potential scenarios for the intersection of two branches. In a transcritical bifurcation (Figure 1(b)), the trivial branch (indicated by a black line) may lose stability when one of the state eigenvalues crosses zero at the branch point, which results in an exchange of stability to the other branch, indicated by a red line. A pitchfork bifurcation (Figure 1(c)) occurs when one solution gives birth to three and often also involves an exchange of stability. 

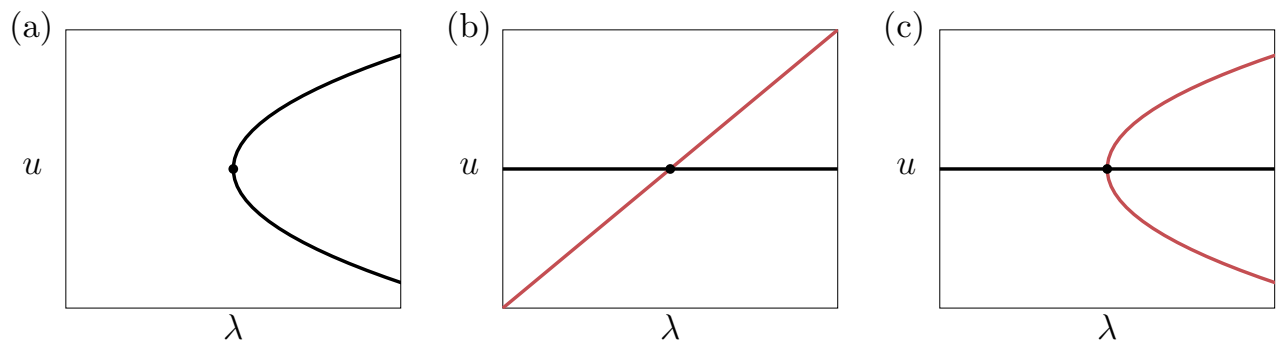

FIG. 1. (a) Fold, (b) transcritical, and (c) pitchfork bifurcations.

The idea of Seydel and Moore and Spence for finding singular solutions to (1.1) is to solve the following extended system (2.1): find $(u, \lambda, \phi) \in U \times \mathbb{R} \times U$ such that

$$
\begin{aligned}
F(u, \lambda) & =0, \\
F_{u}(u, \lambda) \phi & =0, \\
\ell(\phi)-1 & =0,
\end{aligned}
$$

where $\ell: U \rightarrow \mathbb{R}$ is a functional chosen to normalize $\phi$. Equations (2.1b) and (2.1c) imply that $\phi$ is a nonzero eigenfunction associated to the zero eigenvalue of $F_{u}(u, \lambda)$.

Remark 2.2. Several choices of normalization conditions $\ell$ in the Moore-Spence system have been considered $[15,76]$. In this work we set

$$
\ell(\phi)=\|\phi\|_{U}^{2} .
$$

Remark 2.3. In this work, we only consider simple singular points, those for which $\operatorname{dim}\left(\operatorname{ker}\left(F_{u}\left(u^{\star}, \lambda^{\star}\right)\right)\right)=1$. This includes nondegenerate turning points and simple symmetry-breaking pitchfork bifurcations $[49,74]$. We further assume that the solutions of (2.1) are isolated. Bifurcation points of higher multiplicity, satisfying $\operatorname{dim}\left(\operatorname{ker}\left(F_{u}\left(u^{\star}, \lambda^{\star}\right)\right)\right)>1$, also satisfy $(2.1)$ a fortiori but may not be isolated. It is possible to design augmented systems that are selective in the sense that they admit certain kinds of branch points as solutions but not others (e.g., [48, 73]). These typically have the advantage that the solutions are isolated, but the augmented systems become larger and more complicated.

Remark 2.4. Different generalizations have also been studied to compute Hopf bifurcations [67], i.e., points at which a periodic solution arises, in contrast to the stationary solutions considered in this work [33, 40, 41, 60].

Since we are interested in changing the shape of the domain $\Omega$ to control bifurcation diagrams, we now present a shape optimization technique constrained by PDEs.

3. PDE-constrained shape optimization. In this section, we give a brief introduction to PDE-constrained shape optimization. A more detailed exposition of the mathematical and implementation techniques employed here is given in [55, 54].

A shape optimization problem is an optimization problem of the following form: find a domain $\Omega \in \mathcal{U}$ that minimizes a shape functional $J: \mathcal{U} \rightarrow \mathbb{R}$, where $\mathcal{U}$ denotes a set of admissible domains. If evaluating the shape functional $J$ on a domain $\Omega$ requires solving a PDE stated on $\Omega$, the shape optimization problem is said to be PDE-constrained. 
There are competing approaches to define the set of admissible domains $\mathcal{U}$, such as the phase-field method $[8,30]$, the level-set method [3, 45], and the moving mesh method $[4,55]$, among others. In this work, we consider the moving mesh method because its neat integration with standard finite element software allows the automation of several key steps in shape optimization [54]. The main idea of the moving mesh method is to construct the set of admissible domains $\mathcal{U}$ by applying diffeomorphisms to an initial domain $\Omega^{0} \subset \mathbb{R}^{d}$, that is,

$$
\mathcal{U}:=\left\{T\left(\Omega^{0}\right) \mid T \in \mathcal{T}\right\},
$$

where $\mathcal{T}$ is the group of bi-Lipschitz isomorphisms on $\mathbb{R}^{d}$; that is, $T \in \mathcal{T}$ and $T^{-1}$ are Lipschitz continuous. In this setting, shape optimization is carried out by constructing a geometric transformation $T$ so that $T\left(\Omega^{0}\right)$ minimizes $J$. This approach is called the moving mesh method because, in its lowest order discretization, this method is equivalent to replacing the initial domain $\Omega^{0}$ with a finite element mesh and optimizing the coordinates of the mesh nodes while retaining the mesh connectivity [55].

Remark 3.1. The set of admissible domains also contains domains that are not Lipschitz. If the shape functional $J$ is constrained to a PDE, then it is common to restrict the domain of definition of $J$ to all elements of $\mathcal{U}$ that are also Lipschitz to ensure that the PDE constraint is well defined. This restriction can be obtained by further assuming, for instance, that the transformations $T$ are continuously differentiable.

Proving that a shape optimization problem admits a solution is not straightforward. The main results rely on compactness arguments and require additional assumptions on the collection of admissible domains $\mathcal{U}$ [2, Chap. 6.2]. Embedding these assumptions into numerical algorithms is extremely challenging, to the point that in practice one contents oneself with finding a sequence of domains along which the shape functional $J$ decreases. In the framework of moving mesh methods, the sequence of domains is modeled via a sequence of transformations that are commonly constructed iteratively. First, one initializes $T^{(0)}$ with the identity transformation, that is, $T^{(0)}(x)=I(x):=x$ for every $x \in \mathbb{R}^{d}$. Then one constructs the subsequent iterates using the composition formula

$$
T^{(k+1)}=\left(I+\delta T^{(k)}\right) \circ T^{(k)}, \quad k \geq 0,
$$

where $\delta T^{(k)}$ is a suitable geometry update. This sequence of iterates corresponds to the sequence of domains $\left\{T^{(k)}\left(\Omega^{0}\right)\right\}$. More specifically, the $(k+1)$ th element $T^{(k)}\left(\Omega^{0}\right)$ of this sequence of domains is obtained by considering the previous domain $T^{(k)}\left(\Omega^{0}\right)$ and by adding to each of its points $x \in T^{(k)}\left(\Omega^{0}\right)$ the corresponding vector $\delta T^{(k)}(x)$.

The geometry updates $\delta T^{(k)}$ can be computed in a standard fashion using derivatives of the function $J$. As an example, in the next paragraph we present a simple steepest descent shape optimization algorithm. More advanced optimization strategies can be adapted to shape optimization in a similar way.

To construct the sequence $\left\{T^{(k)}\right\}$ using a steepest descent algorithm, we compute the update $\delta T^{(k)}$ as the Riesz representative of the negative shape derivative $-d J$ evaluated at $T^{(k)}[53,55]$. This means that $\delta T^{(k)}$ satisfies the equation

$$
\left(\delta T^{(k)}, V\right)_{\mathcal{H}}=-d J\left(T^{(k)}\left(\Omega^{0}\right) ; V\right) \text { for all } V \in \mathcal{H},
$$

where $\mathcal{H}$ is a suitable Hilbert space with inner product $(\cdot, \cdot)_{\mathcal{H}}$ and

$$
d J\left(T\left(\Omega^{0}\right) ; V\right):=\lim _{s \rightarrow 0^{+}} \frac{J\left((I+s V) \circ T\left(\Omega^{0}\right)\right)-J\left(T\left(\Omega^{0}\right)\right)}{s} .
$$


Once $\delta T^{(k)}$ has been computed, the new transformation $T^{(k+1)}$ is obtained using formula (3.1), possibly scaling $\delta T^{(k)}$ by a sufficiently small step length $s \in(0,1)$ to ensure that $T^{(k+1)}$ is bijective [2, Lem. 6.13]. Listing 1 shows how to implement (in Firedrake) one step of the steepest descent method to minimize the shape functional

$$
J(\Omega)=\int_{\Omega} f \mathrm{~d} x
$$

for the specific two-dimensional function $f\left(x_{1}, x_{2}\right)=x_{1}^{2}+\left(3 x_{2} / 2\right)^{2}$. The shape derivative of $J$ is

$$
d J(\Omega ; V)=\int_{\Omega} \nabla f \cdot V+f \nabla \cdot V \mathrm{~d} x
$$

and the optimal solution is the zero-level set of $f$, which in this case is an ellipse with major and minor axes equal to 1 and $2 / 3$, respectively. To compute the Riesz representative, we use piecewise linear Lagrangian finite elements and consider the inner product

$$
(V, W)_{\mathcal{H}}=\int_{\Omega} D V: D W+V \cdot W \mathrm{~d} x
$$

where $D V: D W$ is the Frobenius inner product of the Jacobians of $V$ and $W$. Figure 2 shows the initial mesh and the mesh obtained after one optimization step. A similar code for a more challenging shape functional is given in [34].

LISTING 1

Firedrake implementation of a steepest descent update for a shape optimization problem with a disk domain.

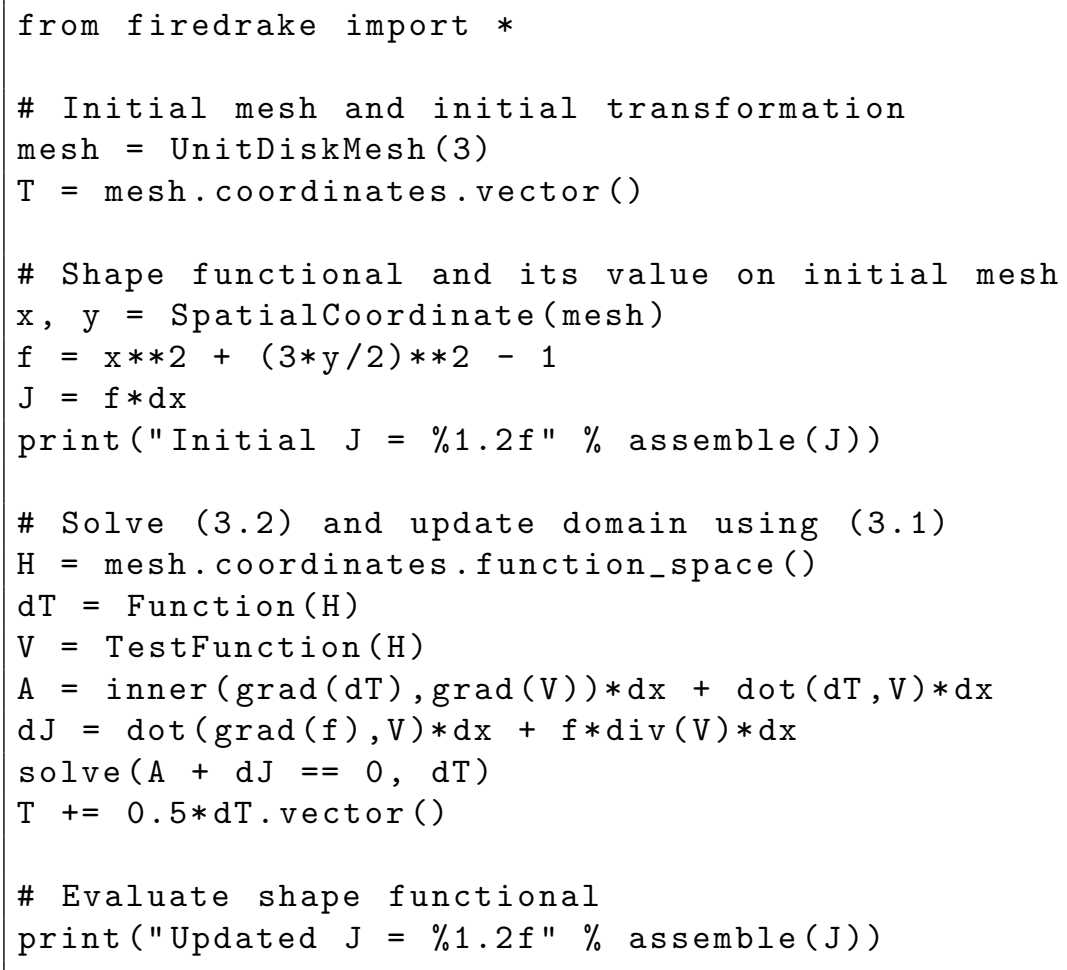

Copyright (c) by SIAM. Unauthorized reproduction of this article is prohibited. 
(a)

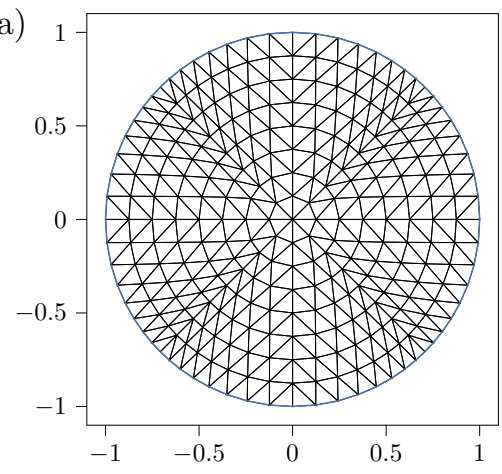

(b)

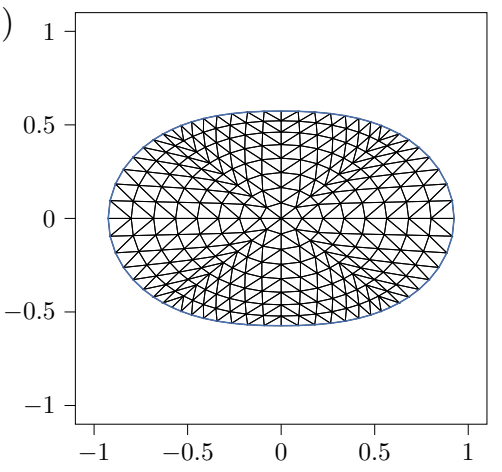

FIG. 2. Initial domain (a) together with the modified domain after one steepest descent update (b) computed using Listing 1 . The optimal solution is an ellipse with major and minor axes equal to 1 and 2/3, respectively. The objective functional $J$ decreases from -0.59 to -1.01 .

Remark 3.2. There are competing requirements on the choice of the Hilbert space $\mathcal{H}$ [55]. On the one hand, it is desirable that the Riesz representative is sufficiently regular to ensure that $T^{(k)}$ is bi-Lipschitz. This suggests using an $H^{d / 2+1}\left(\mathbb{R}^{d}\right)$-type space, which is embedded in $W^{1, \infty}\left(\mathbb{R}^{d}\right)[22$, sect. 5.6.3]. On the other hand, it is desirable that (3.2) can be solved efficiently using standard finite elements, which suggests using an $H^{1}\left(\mathbb{R}^{d}\right)$-type space. In this work, we follow the latter option and employ an inner product based on the linear elasticity equations. This is a standard choice in shape optimization [9,21,61], and, although it is not guaranteed that $\delta T^{(k)} \in W^{1, \infty}(\mathbb{R})$ at the continuous level, its finite element approximation (based on standard Lagrangian finite elements) belongs to $H^{1}(\Omega) \cap W^{1, \infty}(\Omega)$ and is thus suitable to define the transformation $T^{(k)}$.

For the numerical simulations in section 5 we employ the shape optimization software Fireshape [54], which is based on the moving mesh method. This choice offers numerous convenient features, including automated shape differentiation in the Unified Form Language [34], automated adjoint computation via pyadjoint [19, 26], integration with the finite element software Firedrake [58] for efficient linear solvers (e.g., block preconditioners and geometric multigrid) from PETSc [7], and access to numerous optimization algorithms via the Rapid Optimization Library [44], including augmented Lagrangian algorithms [51, Chap. 17.3] to incorporate additional equality (and inequality) constraints such as volume or surface area constraints [54, sect. 2 and 3].

4. Optimization of isolated branch points. In light of sections 2 and 3 , we formulate the problem of finding a shape of the domain $\Omega^{\star}$ such that the operator $F$ admits a branch point $\left(u^{\star}, \lambda^{\star}\right)$ at a target bifurcation parameter $\lambda^{\star} \in \mathbb{R}$ as follows:

$$
\min _{\Omega \in \mathcal{U}} J(\Omega):=\left(\lambda-\lambda^{\star}\right)^{2} \quad \text { subject to } \begin{cases}F(u, \lambda) & =0 \\ F_{u}(u, \lambda) \phi & =0 \\ \|\phi\|_{U}^{2}-1 & =0 .\end{cases}
$$

In this section, we describe an algorithm (Algorithm 4.1) for solving (4.1) and controlling branch points of nonlinear problems of the form of (1.1). 


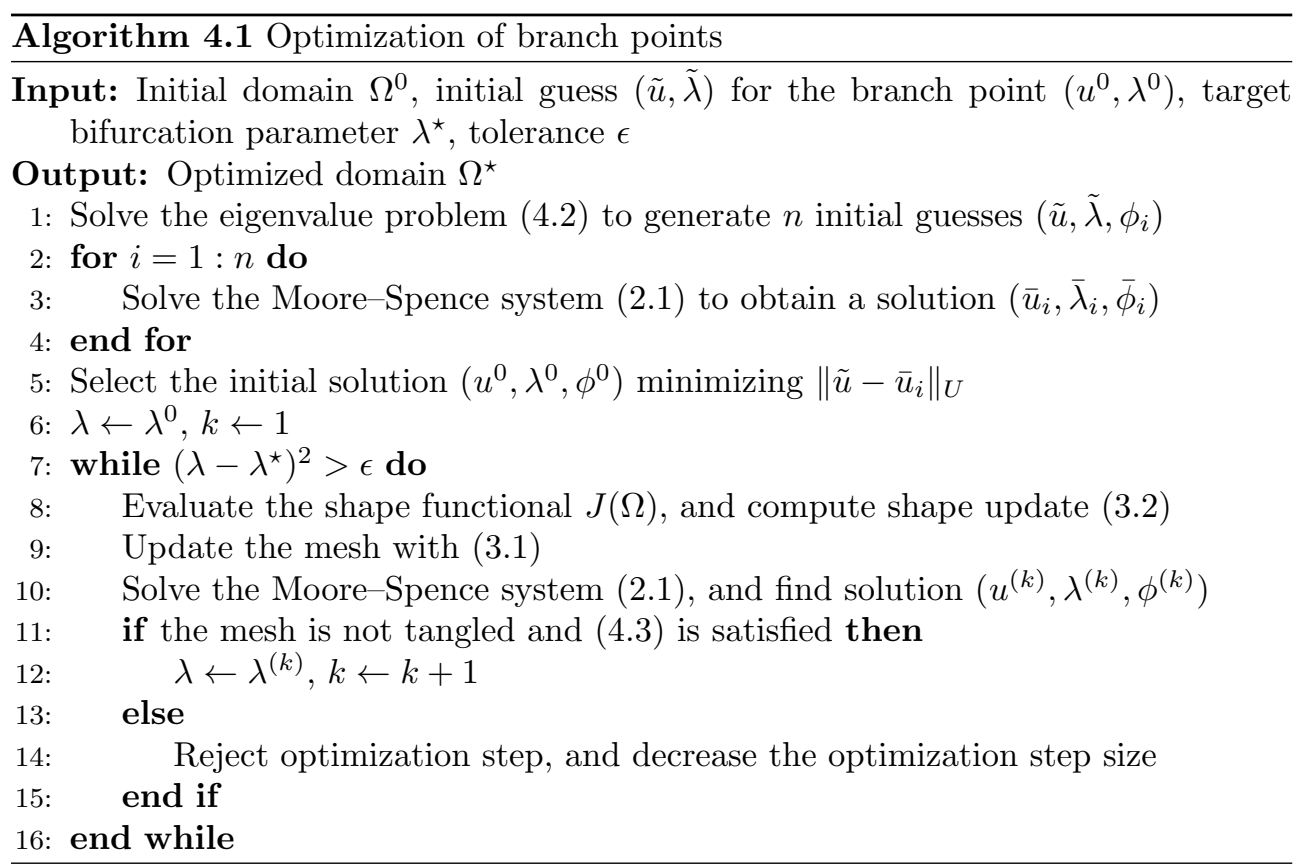

Compared to classical PDE-constrained shape optimization, the problem (4.1) presents an additional difficulty: the state constraint admits multiple solutions by design. More precisely, unless the bifurcation diagram of $F(u, \lambda)=0$ is trivial, there are values of $\lambda$ for which the problem to find $u \in U$ such that $F(u, \lambda)=0$ admits multiple solutions $u$. Additionally, the bifurcation diagram of $F(u, \lambda)=0$ typically includes multiple branch points $(u, \lambda)$ (in the sense of Definition 2.1). For these reasons, to solve (4.1), we must select not only an initial domain $\Omega^{0}$ but also an initial isolated branch point $\left(u^{0}, \lambda^{0}\right)$ that we wish to control. This selection can be performed by first computing the bifurcation diagram of the initial domain $\Omega^{0}$ and selecting $\left(u^{0}, \lambda^{0}\right)$ via visual inspection. Since most algorithms for bifurcation analysis compute branch points approximately, we then refine the approximation of $\left(u^{0}, \lambda^{0}\right)$ using the following procedure. We select a pair $(\tilde{u}, \tilde{\lambda}) \in U \times \mathbb{R}$ from the computed bifurcation diagram that both satisfies $F(\tilde{u}, \tilde{\lambda})=0$ and that is sufficiently close to $\left(u^{0}, \lambda^{0}\right)$. Then, we solve the eigenvalue problem

$$
\text { find }(\mu, \phi) \text { such that } F_{u}(\tilde{u}, \tilde{\lambda}) \phi=\mu \phi
$$

to determine the first $n \in \mathbb{N}$ (a typical value used is $n=5$ ) smallest (single) eigenvalues in magnitude and corresponding eigenfunctions $\phi_{1}, \ldots, \phi_{n}$ (normalized to 1 with respect to the norm $\|\cdot\|_{U}$ ). The eigenvalue problem (4.2) is solved using a Krylov-Schur algorithm with a shift-and-invert spectral transformation [66], implemented in the SLEPc library [38]. Finally, we form $n$ initial guesses $\left(\tilde{u}, \tilde{\lambda}, \phi_{i}\right)$ and solve the Moore-Spence system with Newton's method. This generates a set of (at most) $n$ distinct solutions $\left(\bar{u}_{i}, \bar{\lambda}_{i}, \bar{\phi}_{i}\right)$, from which we finally select the tuple minimizing the difference between $\bar{u}_{i}$ and $\tilde{u}$ in the $U$-norm as the starting point for shape optimization, that is, as $\left(u^{0}, \lambda^{0}, \phi^{0}\right)$.

Remark 4.1. Using this procedure ensures that the initial state $\left(u^{0}, \lambda^{0}, \phi^{0}\right)$ is feasible. In practice, selecting $n>1$ is important because situations like the one 


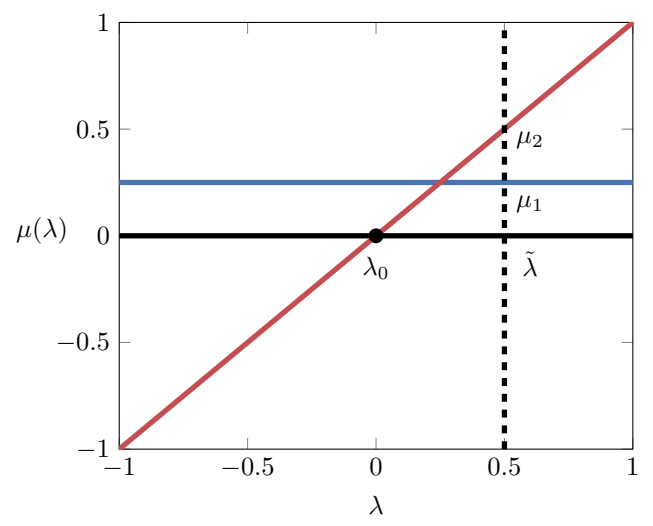

FIG. 3. Schematic of a situation where the eigenfunction associated with the second largest eigenvalue is a better initial guess than the smallest one for the Moore-Spence system. Two eigenvalue paths $\mu_{1}(\lambda)$ (blue) and $\mu_{2}(\lambda)$ (red) are plotted as a function of the parameter $\lambda$. At the solution of the Moore-Spence system $\lambda=\lambda_{0}, 0=\mu_{2}<\mu_{1}$, but the initial guess employed $\lambda=\tilde{\lambda}, \mu_{2}>\mu_{1}$. One would therefore expect the second lowest eigenfunction at $\lambda=\tilde{\lambda}$ to be a better initial guess.

illustrated in Figure 3 might arise; the eigenfunction associated with the lowest eigenvalue for $\tilde{\lambda} \neq \lambda^{0}$ may not be a good guess for the lowest eigenfunction at $\lambda^{0}$ since the eigenvalues may swap order.

Once $\Omega^{0}$ and $\left(u^{0}, \lambda^{0}, \phi^{0}\right)$ have been determined, we use Fireshape [54] to solve (4.1) iteratively with the trust-region optimization algorithm [16] and the inner solver limited memory BFGS [46] implemented in the Rapid Optimization Library [44]. To ensure that the computational mesh is not tangled, we reject optimization steps for which the minimum of the determinant of the Jacobian of the transformation $T$ defined in (3.1) becomes negative. Additionally, we need to ensure that, after a domain update, Newton's method converges to a solution $u^{(k+1)}$ to the Moore-Spence system that lives on the same branch as the one computed at the previous iteration, i.e., is close enough to the solution $u^{(k)}$ obtained in the previous iteration. Hence, undesired branch-switching, caused by multiple solutions to the state equation, might occur during the shape optimization algorithm as observed in [75]. To handle this, we reject domain updates that do not satisfy the inequality

$$
\left\|u^{(k)} \circ\left(I+\delta T^{(k)}\right)^{-1}-u^{(k+1)}\right\|_{U} \leq C\left\|u^{(k+1)}\right\|_{U},
$$

where $I+\delta T^{(k)}$ is the diffeomorphism that updates the domain geometry in the $k$ th iteration and $C$ is an arbitrary constant $(C=0.1$ in subsections 5.1 and $5.3, C=1$ in subsection 5.2).

5. Numerical examples. In this section, we apply the shape optimization strategy described in section 4 to three test cases based on three different differential equations $F$ : the Allen-Cahn equation, the Navier-Stokes equations, and the hyperelasticity equations. The bifurcation diagrams displayed in this section are computed with deflated continuation $[23,24]$, complemented with arclength continuation. These techniques have been successfully applied to a wide range of physical problems such as the deformation of a hyperelastic beam [25], liquid crystals [20, 78], Bose-Einstein condensates $[10,13,14]$, and fluid dynamics [11]. However, our optimization strategy is not tied to a specific algorithm for bifurcation analysis, and other options may be used. 
5.1. Allen-Cahn equation. As the first test case, we consider the cubic-quintic Allen-Cahn equation, which can be used to model phase separation [5, 70]. In strong form, the Allen-Cahn equation is given by

$$
\begin{aligned}
F(u, \lambda):=-0.25 \nabla^{2} u-\lambda u-u^{3}+u^{5} & =0 & & \text { in } \Omega, \\
u & =0 & & \text { on } \partial \Omega .
\end{aligned}
$$

In this example, we consider two-dimensional domains $\Omega \subset \mathbb{R}^{2}$. Therefore, we can set $U=H_{0}^{1}(\Omega)$. We use two different initial domains: a square $\Omega_{\text {square }}$ with rounded corners and edge length 2 (see Figure $6(\mathrm{a})$ ) and a unit disk $\Omega_{\text {disk }}$. The computational meshes of $\Omega_{\text {square }}$ and $\Omega_{\text {disk }}$ are both generated using Gmsh [31] and are composed of 9070 and 13571 triangles, respectively. The Allen-Cahn equation is discretized using piecewise linear continuous Lagrangian finite elements.

In (5.1), several bifurcations occur as $\lambda$ varies. In the left panels of Figures 4 and 5 , we show the bifurcation diagrams computed with deflated continuation [23] for $\lambda \in[0,20]$ when the computational domain is $\Omega_{\text {square }}$ and $\Omega_{\text {disk }}$, respectively. We notice that several branches arise in both cases from the ground state $u=0$. We can order these branches by numbering them from the left. We observe that the solutions $u$ associated to higher branches feature more complicated patterns than the states belonging to the first branches (see Figures 6 and 7). Another interesting observation is the presence of a fold bifurcation on each branch due to the cubic-quintic terms [70].

(a)

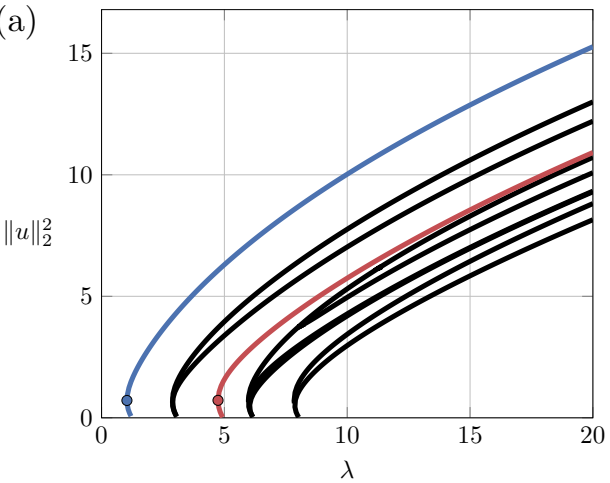

(b)

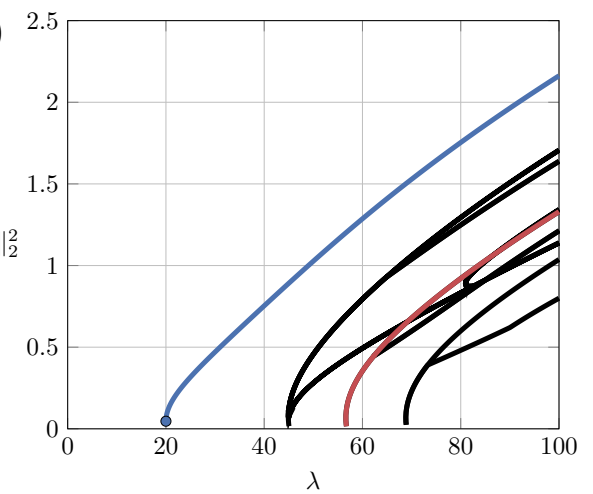

FIG. 4. Bifurcation diagrams of the Allen-Cahn problem defined on a square with round corners $\Omega_{\text {square }}(\mathrm{a})$, together with the diagram after delaying the first bifurcation to $\lambda=20$ (b). The first and third branches are, respectively, highlighted in blue and red.

We now apply the optimization method described in section 4 to delay the birth of the first branch from the ground state in the Allen-Cahn equation to a target bifurcation parameter of $\lambda^{\star}=20$. Here, the whole domain is deformable. In Figures 6 and 7 , we display the evolution of the optimization functional $\mathcal{R}(\lambda)=\left(\lambda-\lambda^{\star}\right)^{2}$ with respect to the trust-region iterations. We also show some snapshots of the solution $u$ and domain $T\left(\Omega^{0}\right)$ for certain values of $\lambda$. We observe that the optimization algorithm reduces the value of the objective functional $\mathcal{R}$ to less than $10^{-11}$ (Figures 6 and 7 ) in about 20 optimization steps.

To verify that the returned solutions satisfy the original objective, we recompute the bifurcation diagrams (using deflated and arclength continuation) on the optimized shapes. The right panels of Figures 4 and 5 illustrate the resulting bifurcation diagrams using the optimized shapes, starting from $\Omega^{0}=\Omega_{\text {square }}$ and $\Omega^{0}=\Omega_{\text {disk }}$, respectively. In both cases, we observe that the bifurcation structure is controlled in the 
(a)

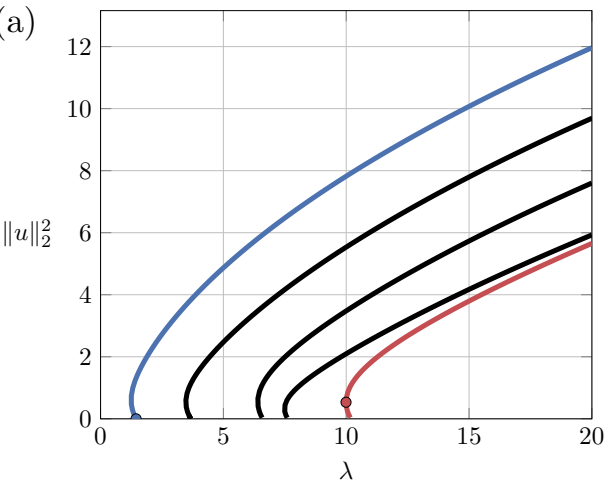

(b)

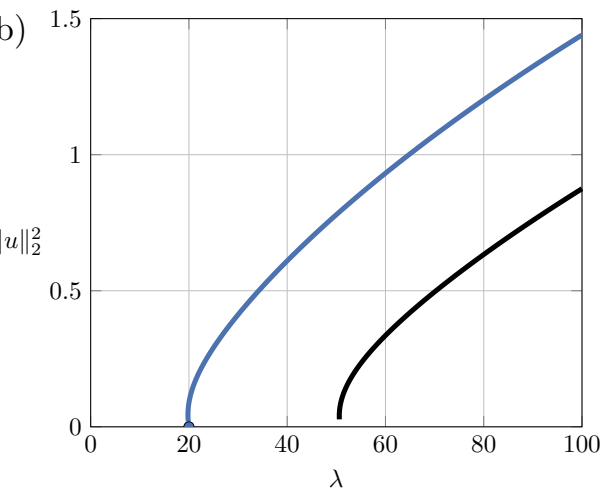

FIG. 5. Bifurcation diagrams of the Allen-Cahn problem defined on the unit disk $\Omega_{\text {disk }}$ (a), together with the diagram after delaying the first bifurcation to $\lambda=20$ (b). The first and fifth branches are, respectively, highlighted in blue and red.

desired way and that the first branch arises at the desirable value of the bifurcation parameter. As expected, the new shape also affects the birth of the later branches. For instance, the second branch of the diagram with $\Omega_{\text {disk }}$ originally arises at $\lambda \approx 4$ and gets delayed to $\lambda \approx 50$ in Figure 5, while the higher branches are still not visible in the range $\lambda \in[0,100]$.

(a)

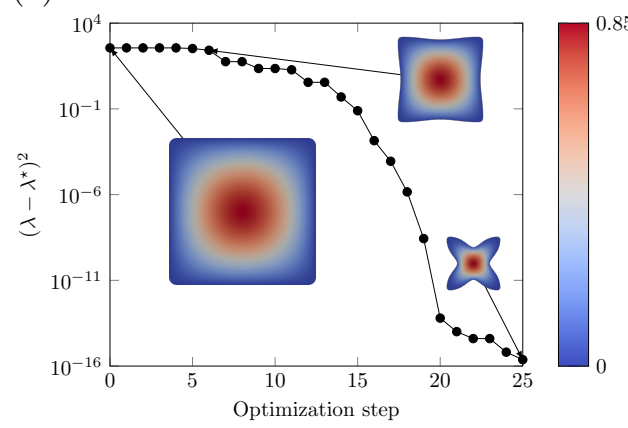

(b)

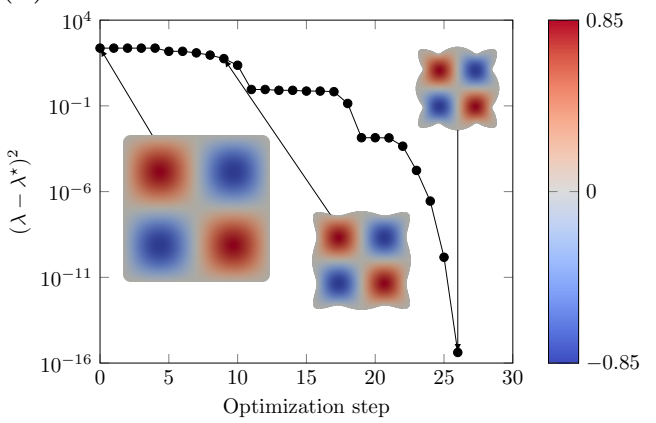

FIG. 6. Evolution of the domain during the shape optimization of the first (a) and third (b) branches (highlighted in blue and red in Figure 4(a)) to the Allen-Cahn equation with a target branch point of $\lambda^{\star}=20$, initialized with the square domain $\Omega_{\text {square. }}$. The curve represents the value of the objective function $\left(\lambda-\lambda^{\star}\right)^{2}$ at each optimization step of the algorithm. The domains are plotted to the same scale.

This example also showcases the complexity and nonlinearity of the problem studied in this work. None of the problems at any level have a unique solution: the PDE may have multiple solutions for a given bifurcation parameter $\lambda$; the MooreSpence system has multiple solutions due to the presence of several branch points; and the shape optimization problem admits multiple solutions, as found from different initial shapes. The shapes displayed in Figure 6(a) and Figure 7(a) are both solutions to the same shape optimization problem but initialized using $\Omega_{\text {square }}$ and $\Omega_{\text {disk }}$.

Finally, we demonstrate that the same approach can be employed to control arbitrary branches. We repeat the previous experiment and shape optimize $\Omega_{\text {square }}$ to postpone the birth of the third branch (which arises near $\lambda=5$; see the left panel of 
A68

(a)

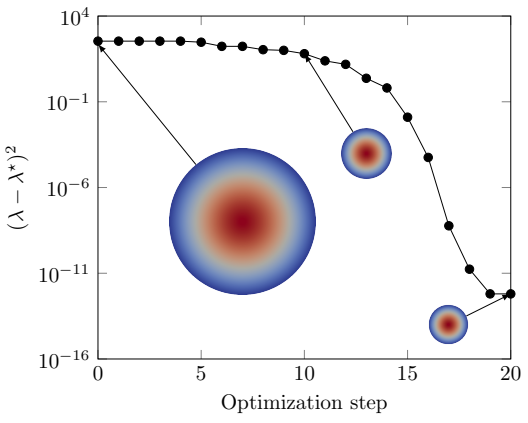

(b)

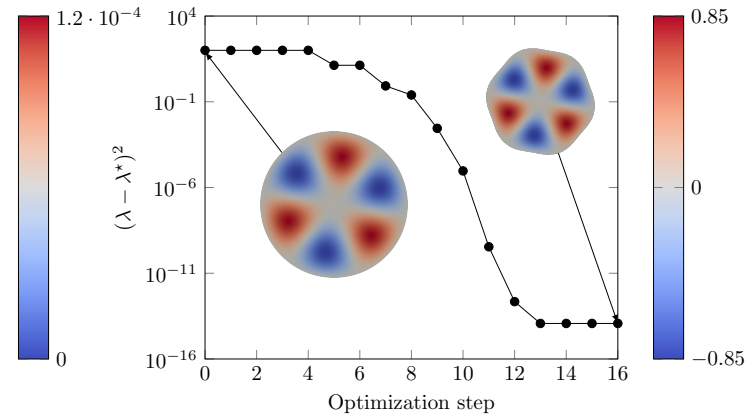

FIG. 7. Evolution of the domain during the shape optimization of the first (a) and fifth (b) branches (highlighted in blue and red in Figure 5(a)) to the Allen-Cahn equation with a target branch point of $\lambda^{\star}=20$, initialized with the disk domain $\Omega_{\text {disk. }}$. The curve represents the value of the objective function $\left(\lambda-\lambda^{\star}\right)^{2}$ at each optimization step of the algorithm. The domains are plotted to the same scale.

Figure 4) to $\lambda^{\star}=20$. In Figure $6(\mathrm{~b})$, we observe that in the shapes obtained by this procedure, the value of $\mathcal{R}$ falls below machine precision in roughly 26 optimization steps. Similarly, in Figure 7(b) we show the optimization history when delaying the birth of the fifth bifurcation of $\Omega_{\text {disk }}$ from (roughly) $\lambda=10$ to $\lambda^{\star}=20$. In this case, the optimized shape resembles a (smoothened) hexagon with edges at the extrema of the function $u$ (indicated by blue and red colors).

5.2. Navier-Stokes equations. In the second test case, we consider the functional $F$ associated to the nondimensionalized steady incompressible Navier-Stokes equations. These read

$$
\begin{aligned}
-\nabla \cdot\left(\frac{2}{\operatorname{Re}} \epsilon(u)\right)+u \cdot \nabla u+\nabla p & =0 & & \text { in } \Omega, \\
\nabla \cdot u & =0 & & \text { in } \Omega, \\
u & =0 & & \text { on } \partial \Omega \backslash\left(\Gamma_{\text {in }} \cup \Gamma_{\text {out }}\right), \\
u & =g & & \text { on } \Gamma_{\text {in }}, \\
p \vec{n}-\frac{2}{\operatorname{Re}} \epsilon(u) \cdot \vec{n} & =0 & & \text { on } \Gamma_{\text {out }},
\end{aligned}
$$

where $u$ is the fluid velocity, $\epsilon(u)=\frac{1}{2}\left(\nabla u+\nabla u^{\top}\right), p$ is the pressure, and Re is the Reynolds number, which in this example plays the role of bifurcation parameter. In particular, we consider an initial geometry formed by the union of two rectangles, $\Omega=[0,2.5] \times[-1,1] \cup[2.5,150] \times[-6,6]$, which represents a channel subject to sudden expansion. The inflow is modeled with a Poiseuille flow $g(x, y)=\left(1-y^{2}, 0\right)^{\top}$ at the leftmost inlet boundary $\Gamma_{\mathrm{in}}$, whereas an outflow boundary condition is imposed on the rightmost outlet boundary $\Gamma_{\text {out }}$. The remaining parts of the boundary carry noslip boundary conditions. The velocity and pressure are discretized with the inf-sup stable Taylor-Hood finite element, i.e., the vector-valued piecewise quadratic continuous Lagrangian element for velocity and the piecewise linear continuous Lagrangian element for pressure.

It is well known that this problem admits multiple solutions depending on the values of the bifurcation parameter Re [27]. The bifurcation diagram for the initial mesh is shown in Figure 8(a). The birth of the first bifurcation from the symmetric 

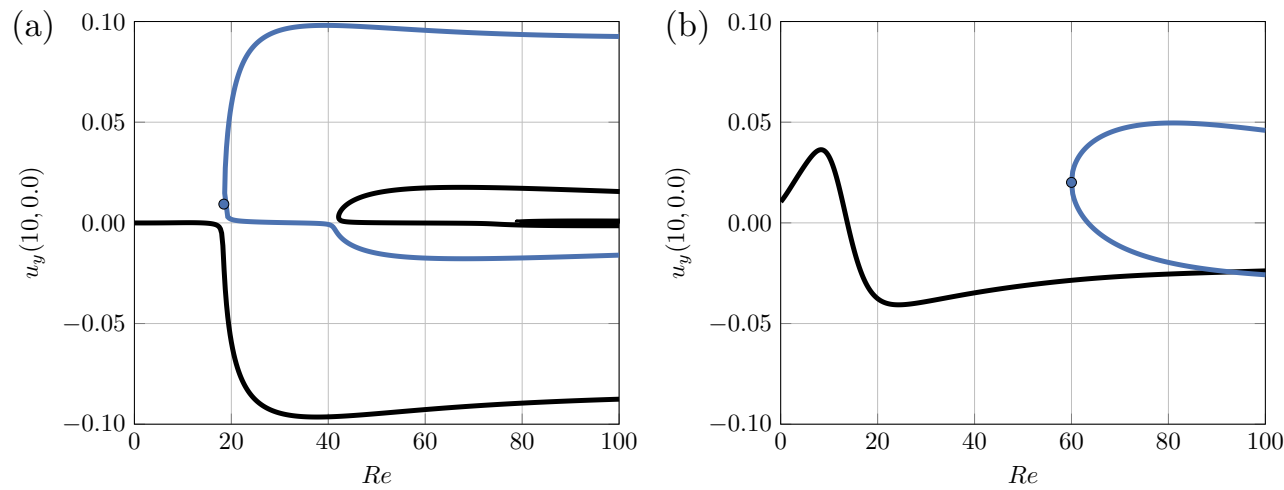

FIG. 8. Left: Initial bifurcation diagram of the Navier-Stokes equations defined on the channel domain $\Omega=[0,2.5] \times[-1,1] \cup[2.5,150] \times[-6,6]$. Right: Bifurcation diagram computed on the optimized channel displayed in Figure 9(c), where the first bifurcation branch (highlighted in blue) arises at $\operatorname{Re}=60$ through a fold bifurcation. The two diagrams are computed with deflated continuation and arclength continuation using $u_{y}(10,0)$ as a diagnostic, where $u_{y}$ is the vertical component of the velocity field $u$.

ground state occurs at $\operatorname{Re} \approx 18.7$. If the mesh were $\mathbb{Z}_{2}$-symmetric about $y=0$, the bifurcation diagram would be symmetric and connected, with branches originating at pitchfork bifurcations from the symmetric ground state. Since symmetry is broken by the use of the unstructured mesh, the pitchforks are perturbed into disconnected fold bifurcations. This phenomenon is very difficult to avoid in practice (especially on complex industrial geometries), but deflation deals with it robustly.

Using the shape optimization approach described in section 4, we modify the shape of the inlet to delay the birth of the first bifurcation from the ground state solution. Since we are interested in changing the shape of the inlet, we fix all boundaries during the shape optimization algorithm except for the following segments:

$$
[(0,1),(2.5,1)], \quad[(2.5,1),(2.5,6)], \quad[(0,-1),(2.5,-1)], \quad[(2.5,-1),(2.5,-6)] .
$$

Figure 9(a) displays the velocity magnitude of the solution on the initial mesh at the bifurcation point located at $R e \approx 18.7$. In Figure $9(\mathrm{~b})-(\mathrm{d})$, we report the shape of the inlet channel after delaying the branch point to $R e=30,60,100$, respectively. We observe that the symmetry of the solution illustrated in Figure 9(a) is broken as we delay the birth of the bifurcation. The shape of the inlet channel becomes bent and forces the fluid to flow downward as the value of the branch point increases from $R e \approx 18.7$ to $R e=100$. In each case, the shape optimization algorithm required between 20 and 30 steps to minimize the loss function to nearly machine precision.

Finally, we apply deflated continuation to compute the bifurcation diagram on the bent domain shown in Figure 9(c), for which the first branch point happens at $R e=60$. The resulting bifurcation diagram is displayed in Figure 8(b). We observe that the first bifurcation branch effectively arises at $R e=60$ through a fold bifurcation and is disconnected from the original solution branch.

5.3. Hyperelastic beam. We consider the shape optimization of a hyperelastic beam under compression. The potential energy of the beam is given by

$$
\Pi(u)=\int_{\Omega} \psi(u) \mathrm{d} x-\int_{\Omega} B \cdot u \mathrm{~d} x
$$


(a)

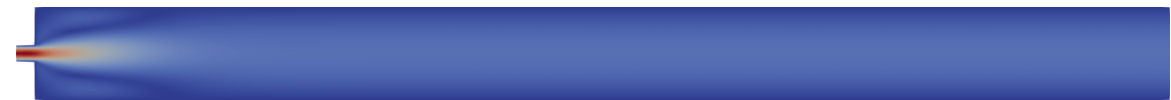

(b)

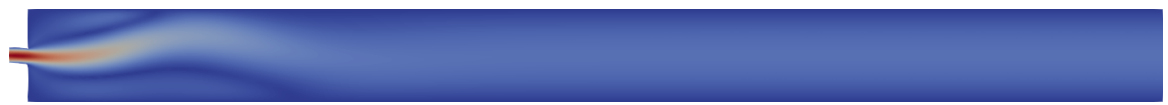

(c)

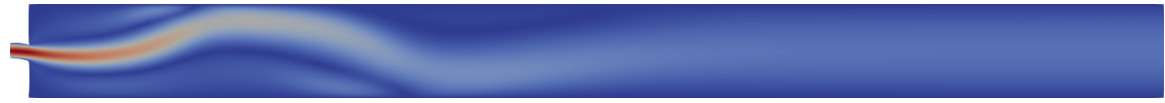

(d)

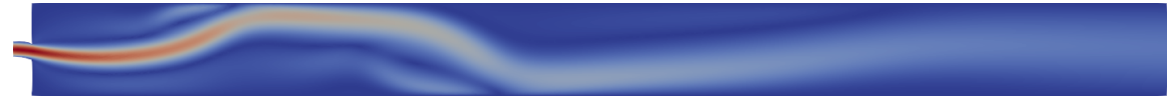

(a)

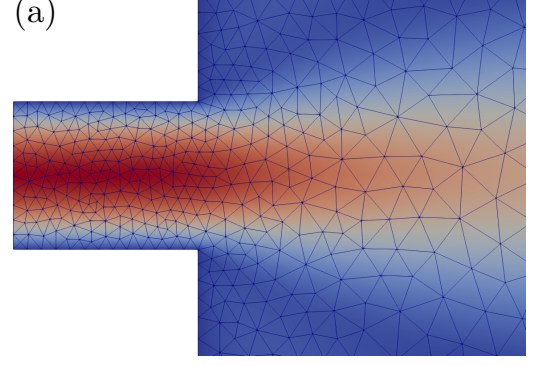

(c)

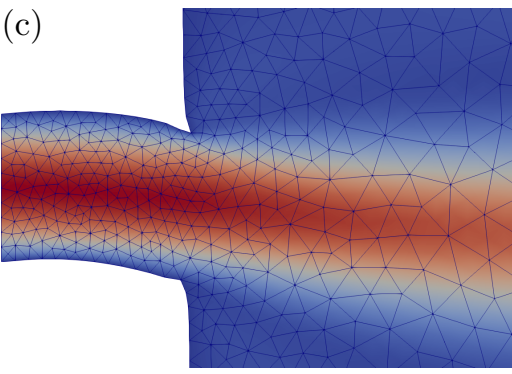

(b)

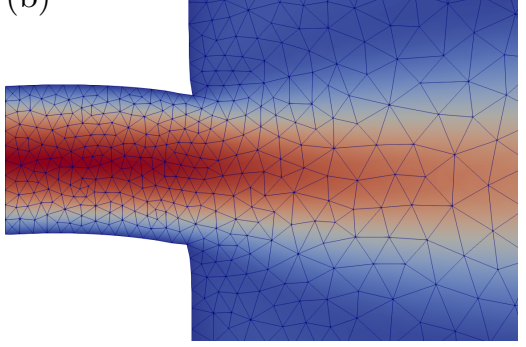

(d)

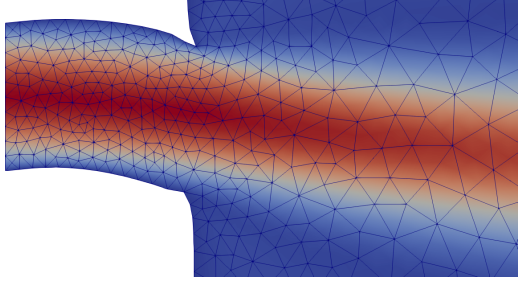

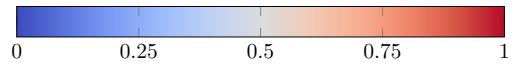

FIG. 9. Velocity magnitude of the solution to the Navier-Stokes equations at the first branch point arising at $\mathrm{Re}=18.7$ (a), 30 (b), 60 (c), 100 (d), together with a magnification of the transformed mesh at the inlet.

where $\Omega=[0,1] \times[0,0.1]$ is the initial domain, $u: \Omega \rightarrow \mathbb{R}^{2}$ is the unknown displacement, $B=(0,-1000)^{\top}$ is a constant body force per unit area, and $\psi$ is a neo-Hookean strain energy density given by

$$
\psi=\frac{\mu}{2}\left(I_{C}-2\right)-\mu \ln (J)+\frac{\lambda}{2} \ln (J)^{2} .
$$

Here $\mu=E /(2(1+\nu)) \approx 3.8 \times 10^{6}$ and $\lambda=E \nu((1+\nu)(1-2 \nu)) \approx 5.8 \times 10^{6}$ denote the Lamé parameters (chosen to yield Young's modulus $E=10^{6}$ and Poisson ratio $\nu=0.3), J=\operatorname{det}(F), F=I+\nabla u, I_{C}=\operatorname{tr}(C)$, and $C=F^{\top} F$. Finally, we complement the potential energy (5.2) with the Dirichlet boundary conditions

$$
u(0, \cdot)=(0,0)^{\top}, \quad u(1, \cdot)=(-\epsilon, 0)^{\top},
$$


where the variable load $\epsilon \in[0,0.2]$ plays the role of bifurcation parameter. On the remaining part of the boundary, denoted by $\Gamma_{N}$, we impose natural traction-free boundary conditions.

Let $n$ denote the outward normal vector and $P(u)$ be the first Piola-Kirchhoff stress tensor, calculated as the derivative of the stored energy density function:

$$
P(u)=\frac{\partial W}{\partial F}=\mu F(\operatorname{tr}(C) I)-\mu F^{\top}+\frac{\lambda}{2} \ln (J) F^{\top},
$$

where $W(F):=\psi(u)$. The boundary value problem considered in this section is

$$
\begin{aligned}
-\nabla \cdot P(u) & =B & & \text { in } \Omega, \\
u & =0 & & \text { on } \Gamma_{L}, \\
u & =(-\epsilon, 0)^{\top} & & \text { on } \Gamma_{R}, \\
P(u) \cdot n & =0 & & \text { on } \Gamma_{N},
\end{aligned}
$$

where $\Gamma_{L}:=\{0\} \times[0,0.1]$ and $\Gamma_{R}:=\{1\} \times[0,0.1]$ denote the left and right boundaries of $\Omega$.
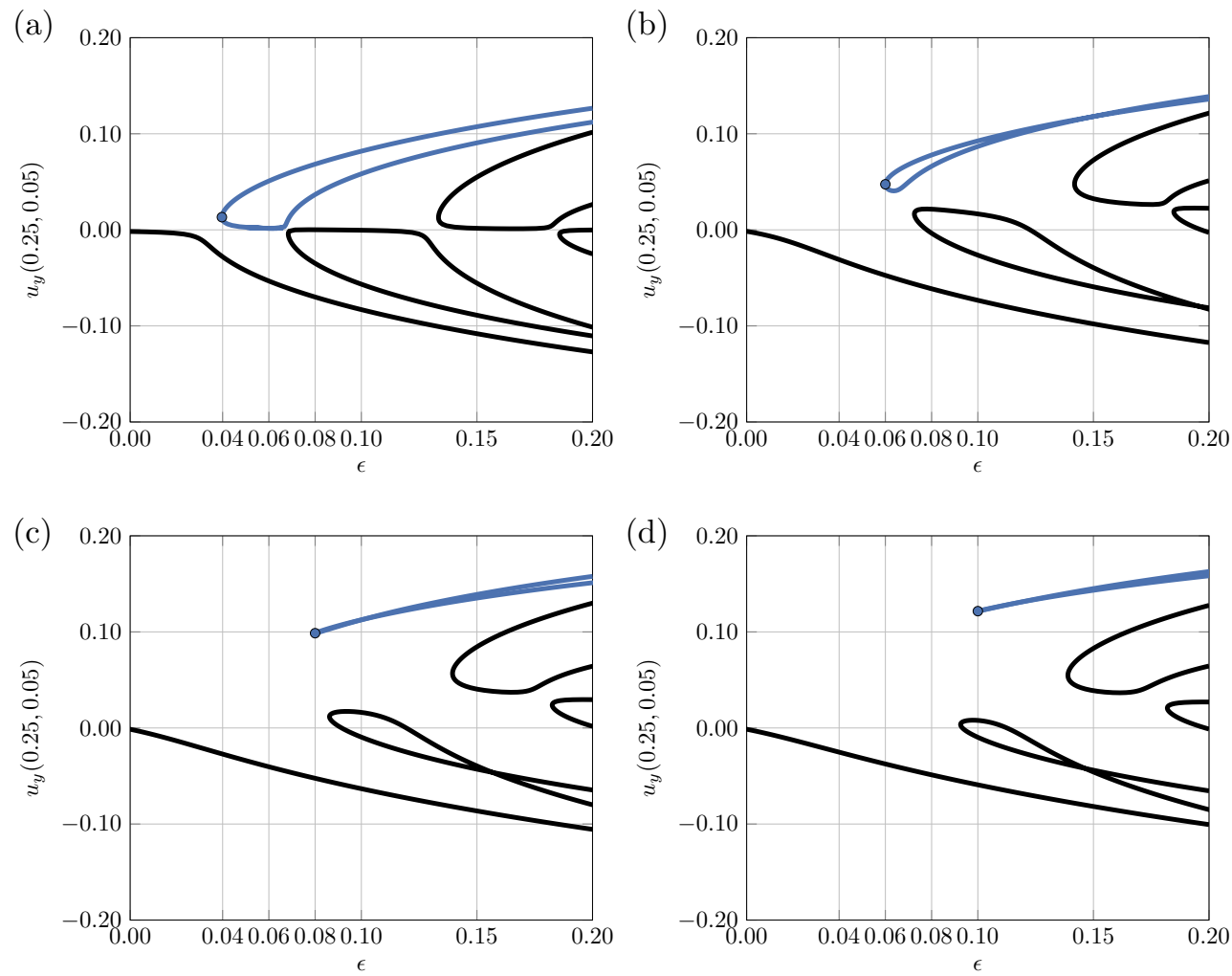

FIG. 10. Bifurcation diagrams of the hyperelasticity problem computed by deflated continuation, where the first fold bifurcation (in the branch highlighted in blue) arises at $\epsilon \approx 0.04$ (original diagram displayed in panel (a)), $\epsilon=0.06$ (b), $\epsilon=0.08$ (c), and $\epsilon=0.1$ (d). The hyperelasticity equations were solved with the different shapes of beam illustrated in Figure 11(a)-(d), respectively. 
The presence of the bifurcation parameter $\epsilon$ in the Dirichlet boundary conditions presents an additional difficulty. Dirichlet boundary conditions such as this are typically enforced in the definition of the trial space by seeking a solution in

$$
\left\{v \in H^{1}\left(\Omega ; \mathbb{R}^{2}\right):\left.v\right|_{\Gamma_{D}}=g(\epsilon)\right\},
$$

where $g(\epsilon)$ is the given boundary data and $\Gamma_{D}=\Gamma_{L} \cup \Gamma_{R}$ is the boundary on which to apply the conditions. However, this approach is not feasible when deriving the Moore-Spence system (2.1) symbolically using the automatic differentiation facilities of Unified Form Language [6], as these require having the bifurcation parameter $\epsilon$ as a variable in the weak form of the equations rather than in the spaces employed.

To circumvent this difficulty, we choose to seek a solution in

$$
V:=\left\{v \in H^{1}\left(\Omega ; \mathbb{R}^{2}\right):\left.v\right|_{\Gamma_{L}}=(0,0)^{\top}\right\}
$$

and to impose the Dirichlet boundary condition on the right boundary $\Gamma_{R}$ using Nitsche's method [29, 50, 64] (as in Xia, Farrell, and Castro [77]). More precisely, we add the terms

$$
\gamma \int_{\Gamma_{R}}(u-g(\epsilon)) \cdot v \mathrm{~d} s-\int_{\Gamma_{R}}(P(u) n) \cdot v \mathrm{~d} s
$$

to the weak formulation arising from the Fréchet differentiation of the hyperelastic energy (5.2). In formula (5.4), $\gamma>0$ is the Nitsche penalization parameter, and, in the numerical experiments, we choose $\gamma=10^{15}$ as in Xia, Farrell, and Castro (justified in [77, App. A]). The final weak form is to find $u \in V$ such that

$$
\Pi^{\prime}(u ; v)+\gamma \int_{\Gamma_{R}}(u-g(\epsilon)) \cdot v \mathrm{~d} s-\int_{\Gamma_{R}}(P(u) n) \cdot v \mathrm{~d} s=0
$$

for all $v \in V$. We discretize (5.5) using piecewise linear continuous Lagrangian finite elements.

We observe several fold bifurcations as the bifurcation parameter $\epsilon$ varies in the interval $[0,0.2]$ on the initial domain. The bifurcation diagram of this problem is illustrated in Figure 10(a) using the diagnostic $u_{y}(0.25,0.05)$ (i.e., the second component of the displacement) to distinguish the different bifurcation branches. In this problem the $\mathbb{Z}_{2}$-symmetry is broken by the nonzero gravitational body force $B$, causing the structure to prefer to buckle downwards rather than upwards. We seek to delay the birth of the first fold bifurcation arising at $\epsilon \approx 0.04$ to $\epsilon=0.06,0.08,0.1$ (see Figure 10(a) and the corresponding displacement $u$ illustrated in Figure 11(a)). In this example, the left and right boundaries of the domain are fixed during the shape optimization. As in subsection 5.2, the shape optimization algorithm required between 20 and 30 steps to minimize the loss function to nearly machine precision in each case.

In Figure 11(b)-(d), we report the shapes returned by the shape optimization procedure and the magnitude of the displacement at the branch points (respectively, arising at $\epsilon=0.06,0.08,0.1)$. We observe that the shape of the beam is bent downward, which makes it difficult to buckle and therefore delays the location of the first fold bifurcation. The bifurcation diagrams of the hyperelasticity equations solved on these new shapes via deflated continuation are displayed in Figure 10(c)-(d). We find that the bifurcation structure of the problem changes as the location of the fold bifurcation is delayed and as the shape of the beam is bent. In Figure 10(d), we see that the branch that we chose to control does indeed arise at $\epsilon=0.1$ as desired but 
(a)

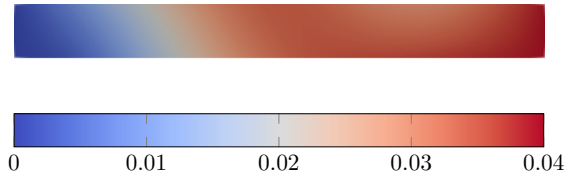

(c)

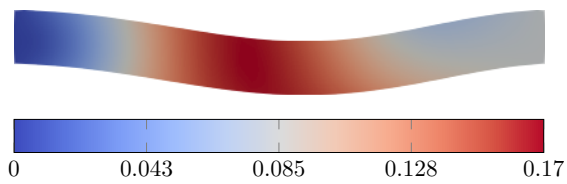

(b)

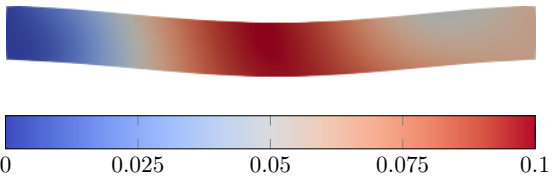

(d)

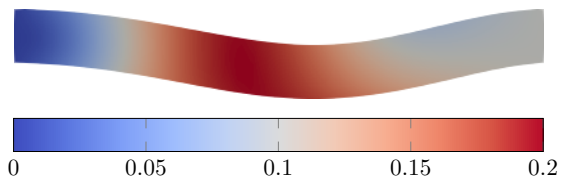

FIG. 11. Magnitude of the displacement $u$ at the first fold bifurcation at $\epsilon=0.04$ (a), $\epsilon=0.06$ (b), $\epsilon=0.08$ (c), and $\epsilon=0.1$ (d) on the respective shapes obtained by the optimization algorithm described in section 4 .

that another fold bifurcation now exists for a smaller bifurcation parameter. This is because the numerical technique described in this paper controls a specific branch, but the other branch points are also modified as the shape of the domain changes during the optimization procedure.

6. Conclusions. We have presented a robust computational technique to control the location of a specified branch point using shape optimization. The key idea is to describe the branch point using the Moore-Spence system. We applied this procedure to vary branch points in the bifurcation diagrams of the cubic-quintic Allen-Cahn equation, the incompressible Navier-Stokes equations, and a hyperelasticity equation. Potential future extensions of the algorithm include its generalization to control Hopf bifurcations [67] as well as incorporating areas or volume constraints on the domain using augmented Lagrangian optimization algorithms [51, Chap. 17.3]. Finally, we expect that this numerical technique could have a wide range of applications in physics and engineering to control the stability of solutions in areas such as the design of new mechanical metamaterials, structural components, flow devices, liquid crystals, and pipes that promote laminar flow.

Code availability. For reproducibility, we archived the Firedrake components [1] and the code [12] used to produce the numerical examples presented in this paper on Zenodo. Detailed instructions to reproduce the results are also available on GitHub at https://github.com/NBoulle/Shape_Bifurcation.

Acknowledgments. We thank the referees for their detailed reviews and suggestions.

\section{REFERENCES}

[1] Software used in 'Control of bifurcation structures using shape optimization', 2021, https:// doi.org/10.5281/zenodo.5217566.

[2] G. Allaire, Conception optimale de structures, Springer, Berlin, 2007.

[3] G. Allaire, F. Jouve, And A.-M. Toader, A level-set method for shape optimization, C. R. Math. Acad. Sci. Paris, 334 (2002), pp. 1125-1130.

[4] G. Allaire and O. Pantz, Structural optimization with FreeFem++, Struct. Multidiscip. Optim., 32 (2006), pp. 173-181.

[5] S. M. Allen And J. W. Cahn, A microscopic theory for antiphase boundary motion and its application to antiphase domain coarsening, Acta Metall., 27 (1979), pp. 1085-1095. 
[6] M. S. Alnæs, A. LogG, K. B. ØlgaArd, M. E. Rognes, and G. N. Wells, Unified Form Language: A domain-specific language for weak formulations of partial differential equations, ACM Trans. Math. Software, 40 (2014), pp. 9:1-9:37, https://doi.org/10.1145/2566630.

[7] S. Balay, S. Abhyankar, M. Adams, J. Brown, P. Brune, K. Buschelman, L. Dalcin, A. Dener, V. Eijkhout, W. Gropp, D. Karpeyev, D. Kaushik, M. Knepley, O. May, L. Curfman Mcinnes, R. Mills, T. Munson, K. Rupp, P. Sanan, B. Smith, S. Zampini, H. Zhang, and H. Zhang, PETSc Users Manual, Technical report ANL-95/11 - Revision 3.14, Argonne National Laboratory, 2020, https://www.mcs.anl.gov/petsc.

[8] L. Blank, H. Garcke, M. H. Farshbaf-Shaker, and V. Styles, Relating phase field and sharp interface approaches to structural topology optimization, ESAIM Control Optim. Calc. Var., 20 (2014), pp. 1025-1058.

[9] S. Blauth, Nonlinear conjugate gradient methods for PDE constrained shape optimization based on Steklov-Poincaré-type metrics, SIAM J. Optim., 31 (2021), pp. 1658-1689.

[10] N. Boullé, E. G. Charalampidis, P. E. Farrell, and P. G. Kevrekidis, Deflation-based identification of nonlinear excitations of the three-dimensional Gross-Pitaevskii equation, Phys. Rev. A, 102 (2020), 053307, https://doi.org/10.1103/PhysRevA.102.053307.

[11] N. Boullé, V. Dallas, And P. E. FARrell, Bifurcation Analysis of Two-Dimensional Rayleigh-Bénard Convection Using Deflation, preprint, arXiv:2102.10576, 2021, https: //arxiv.org/abs/2102.10576.

[12] N. Boullé, P. E. Farrell, and A. Paganini, Software used in 'Control of bifurcation structures using shape optimization', 2021, https://doi.org/10.5281/zenodo.5235244.

[13] E. G. Charalampidis, N. Boullé, P. E. Farrell, and P. G. Kevrekidis, Bifurcation analysis of stationary solutions of two-dimensional coupled Gross-Pitaevskii equations using deflated continuation, Commun. Nonlinear Sci. Numer. Simul., 87 (2020), 105255.

[14] E. G. Charalampidis, P. G. Kevrekidis, and P. E. Farrell, Computing stationary solutions of the two-dimensional Gross-Pitaevskii equation with deflated continuation, Commun. Nonlinear Sci. Numer. Simul., 54 (2018), pp. 482-499, https://doi.org/10.1016/j.cnsns. 2017.05.024.

[15] K. A. Cliffe AND K. H. Winters, The use of symmetry in bifurcation calculations and its application to the Bénard problem, J. Comput. Phys., 67 (1986), pp. 310-326.

[16] A. R. Conn, N. I. M. Gould, And P. L. Toint, Trust Region Methods, SIAM, Philadelphia, 2000.

[17] M. C. Delfour and J.-P. Zolésio, Shapes and Geometries, 2nd ed., SIAM, Philadelphia, 2011.

[18] J. E. Dennis, M. El-Alem, And M. C. Maciel, A global convergence theory for general trustregion-based algorithms for equality constrained optimization, SIAM J. Optim., 7 (1997), pp. 177-207, https://doi.org/10.1137/s1052623492238881.

[19] J. S. Dokken, S. K. Mitusch, And S. W. Funke, Automatic Shape Derivatives for Transient PDEs in FEniCS and Firedrake, preprint, arXiv:2001.10058, 2020, https://arxiv.org/abs/ 2001.10058

[20] D. B. Emerson, P. E. Farrell, J. H. Adler, S. P. Maclachlan, and T. J. Atherton, Computing equilibrium states of cholesteric liquid crystals in elliptical channels with deflation algorithms, Liq. Cryst., 45 (2018), pp. 341-350.

[21] T. Etling, R. Herzog, E. Lohyza, and G. Wachsmuth, First and second order shape optimization based on restricted mesh deformations, SIAM J. Sci. Comput., 42 (2020), pp. A1200-A1225.

[22] L. C. Evans, Partial Differential Equations, 2nd ed., American Mathematical Society, Providence, RI, 2010.

[23] P. E. Farrell, C. H. Beentjes, and Á. Birkisson, The Computation of Disconnected Bifurcation Diagrams, preprint, arXiv:1603.00809, 2016, https://arxiv.org/abs/1603.00809.

[24] P. E. Farrell, A. Birkisson, And S. W. Funke, Deflation techniques for finding distinct solutions of nonlinear partial differential equations, SIAM J. Sci. Comput., 37 (2015), pp. A2026-A2045, https://doi.org/10.1137/140984798.

[25] P. E. Farrell, M. Croci, and T. M. Surowiec, Deflation for semismooth equations, Optim. Methods Softw., 35 (2019), pp. 1248-1271, https://doi.org/10.1080/10556788.2019. 1613655.

[26] P. E. Farrell, D. A. Ham, S. W. Funke, and M. E. Rognes, Automated derivation of the adjoint of high-level transient finite element programs, SIAM J. Sci. Comput., 35 (2013), pp. C369-C393.

[27] R. M. Fearn, T. Mullin, And K. A. Cliffe, Nonlinear flow phenomena in a symmetric sudden expansion, J. Fluid Mech., 211 (1990), pp. 595-608, https://doi.org/10.1017/ S0022112090001707.

[28] Y. Forterre, J. M. Skotheim, J. Dumais, and L. Mahadevan, How the Venus flytrap snaps, Nature, 433 (2005), pp. 421-425, https://doi.org/10.1038/nature03185.

Copyright (c) by SIAM. Unauthorized reproduction of this article is prohibited. 
[29] J. Freund and R. Stenberg, On weakly imposed boundary conditions for second order problems, in Proceedings of the Ninth International Conference on Finite Elements in Fluids, Venice, Italy, Springer, Berlin, 1995, pp. 327-336.

[30] H. Garcke, C. Hecht, M. Hinze, and C. Kahle, Numerical approximation of phase field based shape and topology optimization for fluids, SIAM J. Sci. Comput., 37 (2015), pp. A1846-A1871.

[31] C. Geuzaine and J.-F. Remacle, Gmsh: A 3-D finite element mesh generator with builtin pre-and post-processing facilities, Int. J. Numer. Methods Engrg., 79 (2009), pp. $1309-1331$.

[32] M. Golubitsky, I. Stewart, and D. G. Schaeffer, Singularities and Groups in Bifurcation Theory: Volume II, Springer, New York, 2012.

[33] A. Griewank and G. Reddien, The calculation of Hopf points by a direct method, IMA J. Numer. Anal., 3 (1983), pp. 295-303.

[34] D. A. Ham, L. Mitchell, A. Paganini, and F. Wechsung, Automated shape differentiation in the Unified Form Language, Struct. Multidiscip. Optim., 60 (2019), pp. 1813-1820.

[35] J. Haslinger And R. A. E. MäkInen, Introduction to Shape Optimization, SIAM, Philadelphia, 2003.

[36] M. Heinkenschloss and D. RIDZal, A matrix-free trust-region SQP method for equality constrained optimization, SIAM J. Optim., 24 (2014), pp. 1507-1541, https://doi.org/10.1137/ 130921738.

[37] M. Heinkenschloss And L. N. Vicente, Analysis of inexact trust-region SQP algorithms, SIAM J. Optim., 12 (2002), pp. 283-302, https://doi.org/10.1137/s1052623499361543.

[38] V. Hernandez, J. E. Roman, And V. Vidal, SLEPc: A scalable and flexible toolkit for the solution of eigenvalue problems, ACM Trans. Math. Software, 31 (2005), pp. 351-362.

[39] M. Hinze, R. Pinnau, M. Ulbrich, and S. Ulbrich, Optimization With PDE Constraints, Math. Model. Theory Appl. 23, Springer, Dordrecht, the Netherlands 2009.

[40] C. P. JACKSON, A finite-element study of the onset of vortex shedding in flow past variously shaped bodies, J. Fluid Mech., 182 (1987), pp. 23-45.

[41] A. D. Jepson, Numerical Hopf Bifurcation, Ph.D. thesis, California Institute of Technology, 1981.

[42] C. Jones, Bistable liquid crystal displays, in Handbook of Visual Display Technology, Springer, Berlin, 2012, pp. 1507-1543, https://doi.org/10.1007/978-3-540-79567-4_92.

[43] D. S. Kamenetskiy, J. E. Bussoletti, C. L. Hilmes, V. Venkatakrishnan, L. B. Wigton, AND F. T. Johnson, Numerical evidence of multiple solutions for the Reynolds-averaged Navier-Stokes equations, AIAA J., 52 (2014), pp. 1686-1698, https://doi.org/10.2514/1. j052676.

[44] D. P. Kouri, D. Ridzal, and G. J. von Winckel, Rapid Optimization Library, Technical report SAND2017-12025PE, Sandia National Laboratories, Albuquerque, NM, 2017.

[45] A. LaUrain, A level set-based structural optimization code using FEniCS, Struct. Multidiscip. Optim., 58 (2018), pp. 1311-1334.

[46] D. C. LiU AND J. NocedAL, On the limited memory BFGS method for large scale optimization, Math. Program., 45 (1989), pp. 503-528.

[47] E. Medina, P. E. Farrell, K. Bertoldi, and C. H. Rycroft, Navigating the landscape of nonlinear mechanical metamaterials for advanced programmability, Phys. Rev. B, 101 (2020), 064101.

[48] G. Moore, The numerical treatment of non-trivial bifurcation points, Numer. Funct. Anal. Optim., 2 (1980), pp. 441-472, https://doi.org/10.1080/01630568008816070.

[49] G. Moore And A. Spence, The calculation of turning points of nonlinear equations, SIAM J. Numer. Anal., 17 (1980), pp. 567-576.

[50] J. Nitsche, Über ein Variationsprinzip zur Lösung von Dirichlet-Problemen bei Verwendung von Teilräumen, die keinen Randbedingungen unterworfen sind, Abh. Math. Semin. Univ. Hambg. 36, Springer, Berlin, 1971, pp. 9-15.

[51] J. Nocedal ANd S. Wright, Numerical Optimization, 2nd ed., Springer, New York, 2006.

[52] P. J. Olver, Applications of Lie Groups to Differential Equations, Springer, New York, 2000.

[53] A. Paganini and R. Hiptmair, Approximate Riesz representatives of shape gradients, in System Modeling and Optimization, L. Bociu, J.-A. Désidéri, and A. Habbal, eds., Springer International Publishing, Cham, Switzerland, 2016, pp. 399-409.

[54] A. Paganini And F. Wechsung, Fireshape: A shape optimization toolbox for Firedrake, Struct. Multidiscip. Optim., 63 (2021), pp. 2553-2569, https://doi.org/10.1007/ s00158-020-02813-y.

[55] A. Paganini, F. Wechsung, and P. E. Farrell, Higher-order moving mesh methods for PDE-constrained shape optimization, SIAM J. Sci. Comput., 40 (2018), pp. A2356-A2382.

Copyright (c) by SIAM. Unauthorized reproduction of this article is prohibited. 
[56] P. Penzler, M. Rumpf, And B. Wirth, A phase-field model for compliance shape optimization in nonlinear elasticity, ESAIM Control Optim. Calc. Var., 18 (2012), pp. 229-258.

[57] F. Pichi, M. Strazzullo, F. Ballarin, and G. Rozza, Driving Bifurcating Parametrized Nonlinear PDEs by Optimal Control Strategies: Application to Navier-Stokes Equations With Model Order Reduction, preprint, arXiv:2010.13506, 2020, https://arxiv.org/abs/ 2010.13506 .

[58] F. Rathgeber, D. A. Ham, L. Mitchell, M. Lange, F. Luporini, A. T. T. McRae, G.-T. Bercea, G. R. Markall, and P. H. J. Kelly, Firedrake: Automating the finite element method by composing abstractions, ACM Trans. Math. Software, 43 (2016), pp. 1-27.

[59] R. Reitinger, K.-U. Bletzinger, and E. Ramm, Shape optimization of buckling sensitive structures, Comput. Syst. Engrg., 5 (1994), pp. 65-75.

[60] D. Roose and V. HLAvaČEK, A direct method for the computation of Hopf bifurcation points, SIAM J. Appl. Math., 45 (1985), pp. 879-894.

[61] V. H. Schulz, M. Siebenborn, And K. Welker, Efficient PDE constrained shape optimization based on Steklov-Poincaré-type metrics, SIAM J. Optim., 26 (2016), pp. 2800-2819.

[62] R. SEYDEL, Numerical computation of branch points in ordinary differential equations, Numer. Math., 32 (1979), pp. 51-68, https://doi.org/10.1007/BF01397649.

[63] R. Seydel, Practical Bifurcation and Stability Analysis, Springer, New York, 2009.

[64] N. Sime And C. R. Wilson, Automatic Weak Imposition of Free Slip Boundary Conditions via Nitsche's Method: Application to Nonlinear Problems in Geodynamics, preprint, arXiv: 2001.10639, 2020, https://arxiv.org/abs/2001.10639.

[65] J. SokoŁowski and J.-P. ZolÉsio, Introduction to Shape Optimization, Springer, Berlin, 1992.

[66] G. W. Stewart, A Krylov-Schur algorithm for large eigenproblems, SIAM J. Matrix Anal. Appl., 23 (2002), pp. 601-614.

[67] S. H. Strogatz, Nonlinear Dynamics and Chaos, CRC Press, Boca Raton, FL, 2018.

[68] C. R. Thomsen, F. Wang, And O. Sigmund, Buckling strength topology optimization of $2 D$ periodic materials based on linearized bifurcation analysis, Comput. Methods Appl. Mech. Engrg., 339 (2018), pp. 115-136.

[69] F. Tröltzsch, Optimal control of partial differential equations: Theory, methods and applications, Grad. Stud. Math. 112, AMS, Providence, RI, 2010.

[70] H. Uecker, D. Wetzel, and J. D. M. Rademacher, pde2path - A Matlab package for continuation and bifurcation in $2 D$ elliptic systems, Numer. Math. Theory Methods Appl., 7 (2014), pp. 58-106.

[71] S. Vidoli AND C. MAURInI, Tristability of thin orthotropic shells with uniform initial curvature, Proc. A, 464 (2008), pp. 2949-2966, https://doi.org/10.1098/rspa.2008.0094.

[72] Y. Wang, E. Ferrer, A. Martínez-Cava, Y. Zheng, and E. Valero, Enhanced stability of flows through contraction channels: Combining shape optimization and linear stability analysis, Phys. Fluids, 31 (2019), 074109.

[73] H. WeBER, On the numerical approximation of secondary bifurcation problems, in Numerical Solution of Nonlinear Equations, E. L. Allgöwer, K. Glashoff, and H.-O. Peitgen, eds., Springer, Berlin, 1981, pp. 407-425.

[74] B. Werner And A. Spence, The computation of symmetry-breaking bifurcation points, SIAM J. Numer. Anal., 21 (1984), pp. 388-399.

[75] J. Williams, F. Wechsung, B. Turney, S. Waters, and D. Moulton, Shape optimisation for faster washout in recirculating flows, J. Fluid Mech., 914 (2021), A37.

[76] K. H. Winters, T. Plesser, And K. A. Cliffe, The onset of convection in a finite container due to surface tension and buoyancy, Phys. D, 29 (1988), pp. 387-401.

[77] J. Xia, P. E. Farrell, And S. G. P. Castro, Nonlinear bifurcation analysis of stiffener profiles via deflation techniques, Thin-Walled Struct., 149 (2020), 106662.

[78] J. Xia, S. Maclachlan, T. J. Atherton, and P. E. Farrell, Structural landscapes in geometrically frustrated smectics, Phys. Rev. Lett., 126 (2021), 177801, https://doi.org/ 10.1103/PhysRevLett.126.177801.

Copyright (c) by SIAM. Unauthorized reproduction of this article is prohibited. 